06

\title{
Расчет параметров источника бифотонного излучения в широком диапазоне длин волн
}

\author{
(С) Г.Г. Левин ${ }^{1}$, А.А. Самойленко , К.Н. Миньков ${ }^{1,2}$, Д.Д. Ружицкая ${ }^{1,3}$ \\ ${ }^{1}$ Всероссийский научно-исследовательский институт оптико-фризических измерений, \\ 119361 Москва, Россия \\ ${ }^{2}$ Московский институт электроники и математики им. А.Н. Тихонова, \\ Национальный исследовательский университет высшей школы экономики, \\ 101000 Москва, Россия \\ ${ }^{3}$ Московский государственный университет им. М.В. Ломоносова, \\ Физический фракультет, \\ 119991 Москва, Россия \\ ฯe-mail: asamoylenko@vniiofi.ru \\ Поступила в редакцию 31.01.2018 г. \\ В окончательной редакции 16.08.2018 г.
}

Разработан конструктивный алгоритм определения параметров нелинейного кристалла для генерации коррелированных фотонов в заданном диапазоне длин волн с помощью эффекта спонтанного параметрического рассеяния. С помощью разработанного метода выполнен расчет параметров кристалла ВВО, способного генерировать бифотонное излучение в диапазоне длин волн от 0.548 до $1.55 \mu \mathrm{m}$ при длине волны накачки $0.405 \mu \mathrm{m}$. Дано описание эксперимента, подтверждающего работоспособность метода.

DOI: $10.21883 /$ OS.2018.12.46942.32-18

\section{Введение}

В настоящее время востребованы и активно развиваются такие направления, как квантовая криптография $[1,2]$, квантовые компьютеры, квантовая телепортация [3]. В частности, уже несколько коммерческих фирм в мире $[4,5]$ изготавливает и устанавливает в организациях системы квантового распределения ключей, функционирование которых основано на принципах квантовой криптографии.

Для исследований в области квантовых информационных технологий требуются источники однофотонного излучения. Эти источники коммерчески доступны [6]. Однако настроены они на определенную длину волны. Тем не менее, большой круг задач, стоящий перед исследователями, требует универсальный источник, способный генерировать однофотонное излучение в широком спектре [7-9].

В настоящей работе рассматривается вероятностный источник однофотонного излучения с объявляющим (heralding) каналом. В обзоре [10] было проведено сравнение однофотонных источников различного типа. С точки зрения приближенности к идеальному однофотонному источнику по критерию корреляционной функции второго порядка $g^{(2)}(0)[10]$ одними из наилучших являются вероятностные источники, основанные на эффекте спонтанного параметрического рассеяния. Такие источники широко применяются в задачах квантовой информатики и криптографии [12].

В качестве источника бифотонного излучения использовался нелинейный кристалл. Его частотно-угловой спектр излучения определяется осью ориентации оси нелинейного кристалла относительно оси накачки. Это приводит к тому, что для каждой конкретной малофотонной системы необходимо создавать свой источник излучения. В целом ряде задач, в частности, при создании измерительных приборов для метрологического обеспечения квантовых информационных систем, необходимо использовать источники бифотонного излучения с заданным спектром. Создание подобных приборов необходимо для определения квантовой эффективности детекторов одиночных фотонов $[10,13,14]$.

Целью настоящей работы является разработка конструктивного алгоритма расчета параметров нелинейного кристалла, который давал бы коррелированные фотоны в заданном диапазоне длин волн. С помощью разработанного алгоритма был проведен расчет параметров нелинейного кристалла с непрерывным спектром бифотонов до $1550 \mathrm{~nm}$ при длине волны накачки $405 \mathrm{~nm}$. Длина волны $1550 \mathrm{~nm}$ представляет интерес, поскольку на этой длине волны находится окно прозрачности плавленого кварца, из-за чего она широко применяется в волоконно-оптических линиях связи. Длина волны накачки была выбрана $405 \mathrm{~nm}$, поскольку, с одной стороны, целесообразно выбирать источник накачки как можно более коротковолновым, а с другой стороны коммерчески доступные более коротковолновые лазеры обладают на порядок более высокой стоимостью.

Длина волны второго фотона в паре при заданных длинах волн накачки и первого фотона определяется из закона сохранения энергии - энергия фотона накачки должна быть равна сумме энергий фотонов в паре. Таким образом, выбирая более коротковолновый лазер для накачки, мы расширяем спектр получаемых фотонов. 


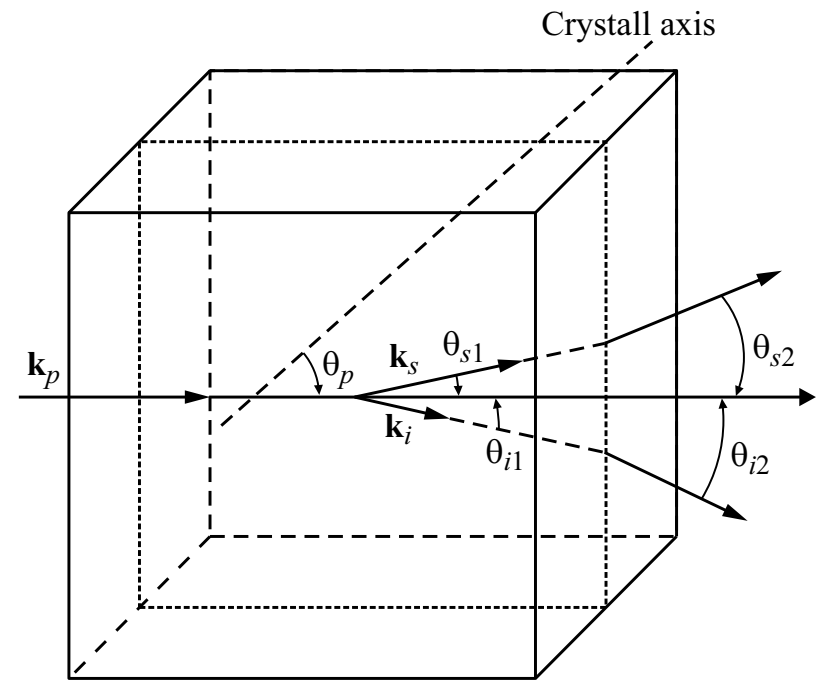

Рис. 1. Иллюстрация эффекта СПР: $\mathbf{k}_{p}, \mathbf{k}_{s}, \mathbf{k}_{i}$ - волновые векторы фотонов накачки, сигнальной, холостой волн соответственно, $\theta_{p}$ - угол наклона оптической оси кристалла к волне накачки, $\theta_{s}, \theta_{i}-$ углы отклонения сигнальной и холостой волн от волны накачки.

\section{Метод определения параметров кристалла}

Эффект спонтанного параметрического рассеяния (СПР) проявляется в нелинейных кристаллах с ненулевым значением $\chi^{(2)}$. Для практических целей используют кристаллы со значительной степенью нелинейности. Эффект заключается в следующем. На нелинейный кристалл падает фотон накачки (pump) с частотой $v_{p}$ и, находясь в нелинейной среде, распадается на два фотона: сигнальный (signal) с частотой $v_{s}$ и холостой (idler) c частотой $v_{i}$. Сигнальная и холостая волны могут иметь одинаковую (такое рассеяние называют СПР, тип I) или различную поляризацию (тип II) и называются в зависимости от целей эксперимента. Схема процесса показана на рис. 1. Данная схема приведена для одноосного кристалла.

Наиболее широко для получения эффекта СПР используются такие нелинейные кристаллы, как ниобат лития $\mathrm{LiNbO}_{3}(\mathrm{NL})$, триборат лития $\mathrm{LiB}_{3} \mathrm{O}_{5}$ (LBO), дигидроортофосфат калия $\mathrm{KH}_{2} \mathrm{PO}_{4}(\mathrm{KDP})$, бета-борат бария $\mathrm{BaB}_{2} \mathrm{O}_{4}(\mathrm{BBO})$. Для задач настоящей работы был выбран кристалл ВВО, поскольку он обладает высоким коэффициентом нелинейности, широкой полосой пропускания, широким диапазоном углов фазового синхронизма, высоким порогом разрушения, большим диапазоном рабочих температур и высокой оптической однородностью [15].

Генерация двух коррелированных фотонов в ходе СПР возможна только при выполнении так называемых условий фазового синхронизма (они могут и не выполняться вообще). Эти условия накладывают определенные требования на соотношения между волной накачки, ориентацией оптических осей кристалла, сигнальной и холостой волнами. Иными словами, генерируемое излучение СПР будет иметь частотно-угловой спектр, однозначно определяемый условиями фазового синхронизма. Этот спектр будет зависеть от длины волны накачки и ориентации осей кристалла.

Таким образом, настоящий метод должен давать ответ на вопрос о том, при каких параметрах кристалла генерируемый частотно-угловой спектр СПР будет обладать требуемыми характеристиками. К параметрам кристалла относятся ориентация осей кристалла, длина волны накачки. К спектру СПР предъявляются следующие требования: диапазон длин волн должен перекрывать требуемый для конкретной задачи; угловой спектр излучения СПР, соответствующий требуемому волновому диапазону, должен удовлетворять геометрическим параметрам конкретной задачи. В части последнего требования обычно необходимо, чтобы излучение СПР имело небольшой угол расходимости относительно луча накачки. В этом случае генерируемые фотоны не перекрываются выходной апертурой кристалла.

При расчете характеристик СПР полагают, что нелинейный кристалл не поглощает энергию и не испытывает отдачу [15]. Процесс СПР возможен только при выполнении условий фазового синхронизма, которые заключается в выполнении законов сохранения энергии и импульса для фотонов накачки, сигнального и холостого [16]:

$$
v_{p}=v_{s}+v_{i},
$$

где $v_{p}, v_{s}, v_{i}$ - частоты фотонов накачки, сигнальной и холостой волн соответственно,

$$
\mathbf{k}_{p}=\mathbf{k}_{s}+\mathbf{k}_{i}
$$

$\mathbf{k}_{p}, \mathbf{k}_{s}, \mathbf{k}_{i}-$ волновые векторы фотонов накачки, сигнальной и холостой волн соответственно. Система уравнений (1) и (2) позволяет определить значения

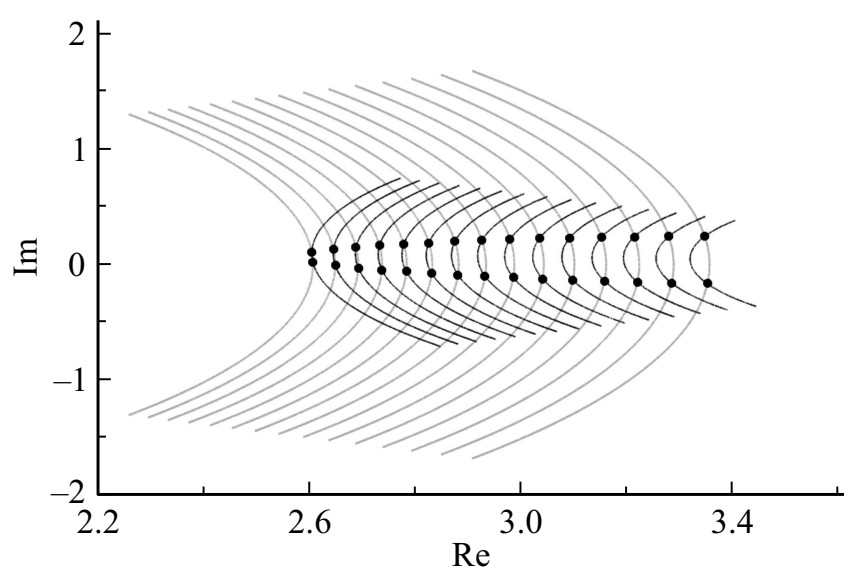

Pис. 2. Полученные проекции волновых векторов $\mathbf{k}_{s}$ (светлые линии) и $\mathbf{k}_{p}-\mathbf{k}_{i}$ (темные линии) на комплексной плоскости при изменении сигнальной длины волны $\lambda_{s}$ и фиксированном значении угла $\theta_{p}$. Черным выделены точки пересечения. 

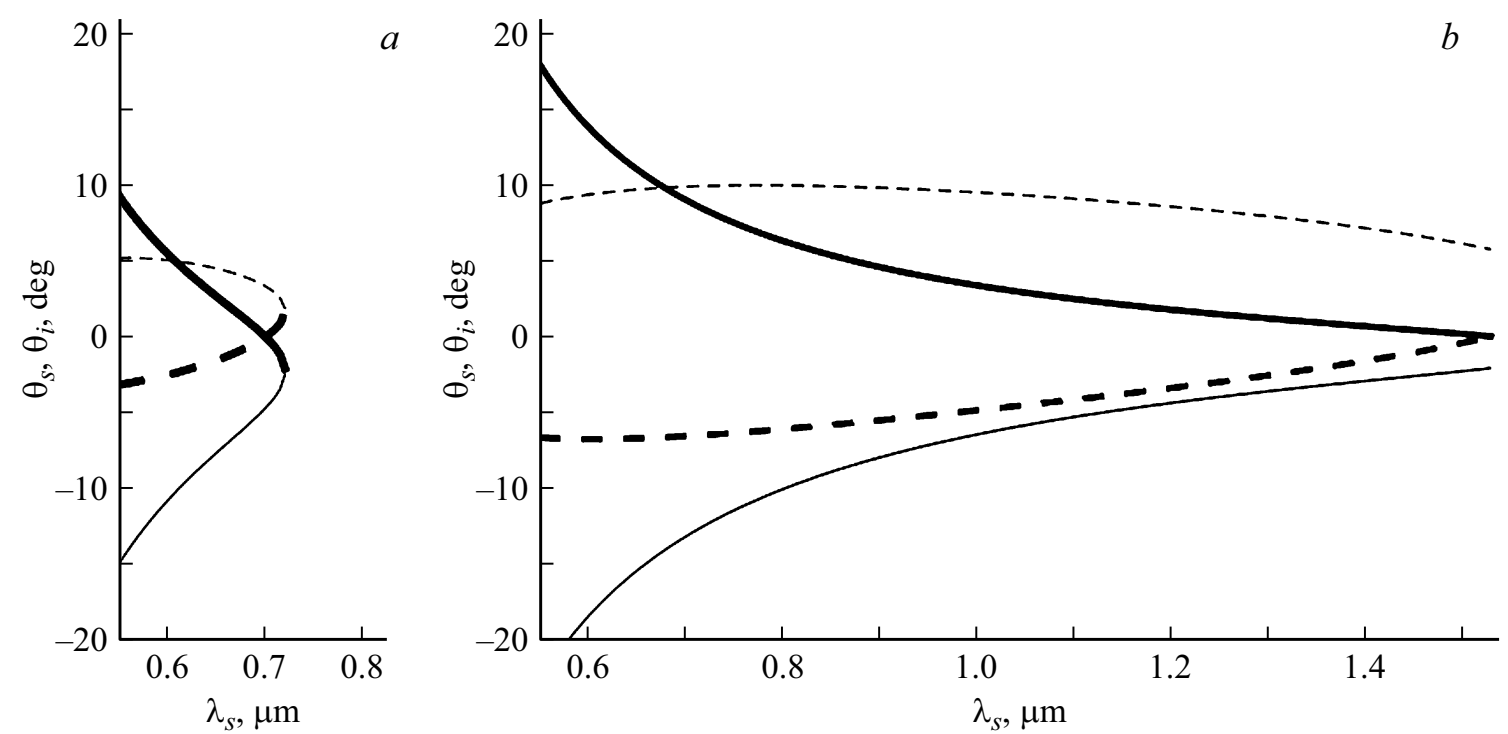

Рис. 3. Зависимость $\theta_{s}\left(\lambda_{s}\right)$ (штриховые линии) и $\theta_{i}\left(\lambda_{s}\right)$ (сплошные линии) для фиксированного значения $\theta_{p}=38^{\circ}(a), 55.5^{\circ}(b)$. Одинаковая толщина линий соответствует парам коррелированных фотонов.

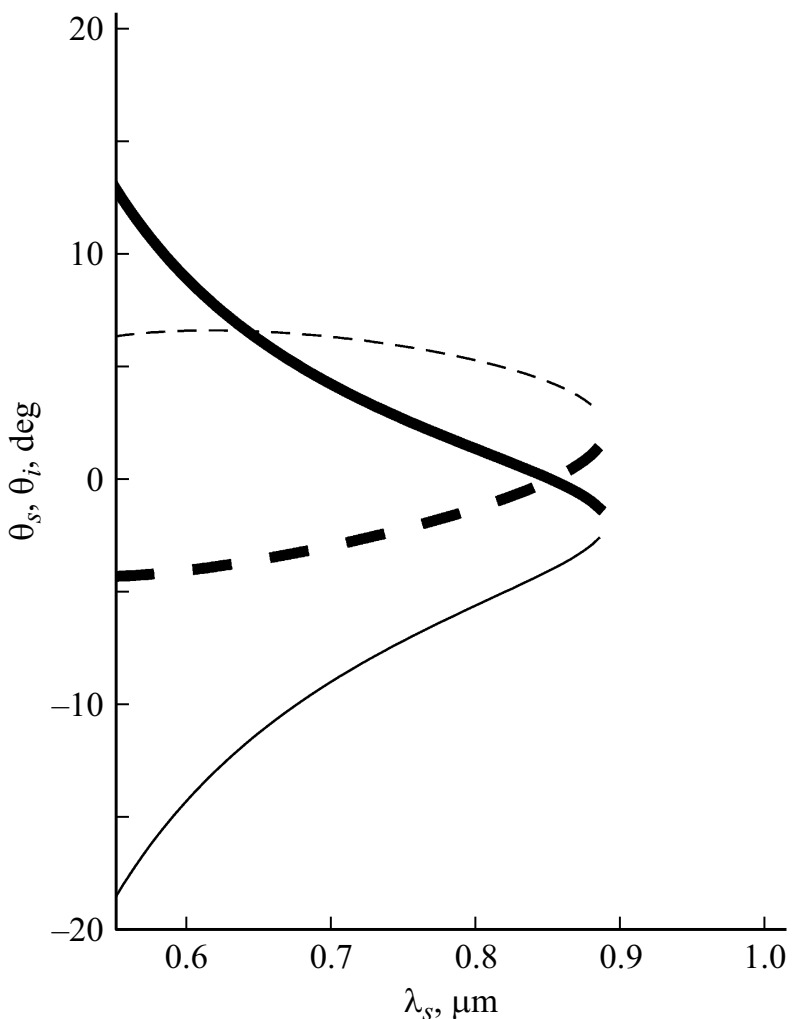

Рис. 4. Зависимость $\theta_{s}\left(\lambda_{s}\right)$ (штриховые линии) и $\theta_{i}\left(\lambda_{s}\right)$ (сплошные линии) для фиксированного значения $\theta_{p}=43^{\circ}$. Одинаковая толщина линий соответствует парам коррелированных фотонов.

углов $\theta_{s}$ и $\theta_{i}$, под которыми будут рассеяны фотоны коррелированной пары при заданных длинах волн накачки и сигнальной.
Для каждого из значений $\lambda_{s}$ можно рассчитать значение $\lambda_{i}$, по формуле, следующей из формулы (1):

$$
\lambda_{i}=\frac{1}{\frac{1}{\lambda_{p}}-\frac{1}{\lambda_{s}}} .
$$

В частности, при длине волны накачки $0.405 \mu \mathrm{m}$ и длине волны одного из фотонов $1.55 \mu \mathrm{m}$, другой фотон пары будет иметь длину волны $0.548 \mu \mathrm{m}$.

Таким образом, задача сводится к решению системы уравнений (1) и (2) и нахождению такого угла $\theta_{p}$, при котором выполняются условия фазового синхронизма для требуемого диапазона длин волн, а также определение соответствующих углов рассеяния коррелированных фотонов. Исходными данными для проводимого расчета являются тип кристалла $-\mathrm{BBO} ; \lambda_{p}-$ длина волны накачки, $0.405 \mu \mathrm{m} ; \theta_{p}$ - диапазон возможных углов, под которыми вырезан кристалл, от 0 до 90; $\lambda_{s}-$ требуемый спектр длин волн, от 0.548 до $1.55 \mu \mathrm{m}$.

Для реальных кристаллов система уравнений (1) и (2) не имеет аналитического решения из-за эффектов дисперсии (зависимость показателя преломления от частоты излучения) и двулучепреломления. Эффект двулучепреломления приводит к тому, что показатель преломления будет зависеть от поляризации и направления распространения света. Для произвольной волны, которая поляризована в плоскости оптической оси кристалла, действует эффективный показатель преломления, который равен [15]

$$
n_{\mathrm{ef}}=\left(\sqrt{\frac{\cos ^{2} \theta}{n_{0}^{2}(\lambda)}+\frac{\sin ^{2} \theta}{n_{e}^{2}(\lambda)}}\right)^{-1},
$$

где $\theta-$ угол между лучом и оптической осью кристалла. Зависимости от длины волны показателей преломления 
$n_{0}$ (обыкновенная волна) и $n_{e}$ (необыкновенная волна) описываются формулами Селмейера [15]. Волна, поляризованная перпендикулярно плоскости оптической оси кристалла, имеет показатель преломления $n_{0}$.

Алгоритм поиска требуемого угла $\theta_{p}$ заключался в последовательном переборе с определенным шагом всех возможных углов от $0^{\circ}$ до $90^{\circ}$ и определении для каждого значения $\theta_{p}$ спектра СПР в заданном диапазоне. При заданном $\theta_{p}$ для каждого значения $\lambda_{s}$ в требуемом диапазоне с определенным шагом проверялись условия фазового синхронизма. Если условия фазового синхронизма выполнялись, полученные значения $\lambda_{i}, \theta_{s}$ и $\theta_{i}$ записывались в файл.

Проверка выполнения условий фазового синхронизма для заданных $\theta_{p}$ и $\lambda_{s}$ производилась только в плоскости оптической оси кристалла. Данная плоскость представляет практический интерес, поскольку наиболее удобна для экспериментальной реализации оптической схемы. Решение уравнений фазового синхронизма находилось следующим образом. В плоскости волновых векторов строились две кривые: $k_{s}\left(\theta_{s}^{l}\right)$ и $\left[k_{p}-k_{i}\right]\left(\theta_{i}^{m}\right)$, где индексы $l$ и $m$ соответствуют разным значениям углов $\theta_{s, i}$ в диапазоне от $-20^{\circ}$ до $20^{\circ}$ с определенным шагом. Полученные проекции показаны на рис. 2. Наличие пересечения векторов соответствует выполнению условия фазового синхронизма. Если полученные кривые не имеют точек пересечения, то условие фазового синхронизма считается невыполненным. Значения $\theta_{s}$ и $\theta_{i}$ в точках пересечения соответствуют направлениям вылета фотонов. Значения шагов перебора параметров в приведенном выше алгоритме подбирались такими, чтобы при дальнейшем уменьшении шагов результаты расчетов не изменялись с погрешностью $0.1 \%$. Вычислительной мощности современного настольного компьютера при этом оказалось достаточно для завершения вычислений в течение трех часов.

На рис. 3 приведены некоторые примеры зависимостей $\theta_{s}\left(\lambda_{s}\right)$ и $\theta_{i}\left(\lambda_{s}\right)$ для разных углов $\theta_{p}$. По данному графику можно определить углы наблюдения $\theta_{s}$ и $\theta_{i}$ генерируемых фотонов в зависимости от длины сигнальной волны $\lambda_{s}$. Одинаковая толщина линий на графике показывает пары коррелированных фотонов. Полученный набор графиков позволяет определить, какие длины волн генерируемых фотонов будут наблюдаться при выбранном угле $\theta_{p}$ оптической оси кристалла. Так, при $\theta_{p}=38^{\circ}$ (рис. 3,a) наблюдается спектр сигнальной волны от 0.548 до $0.73 \mu \mathrm{m}$ и спектр холостой волны от 0.9 до $1.25 \mu \mathrm{m}$. Дополнительно можно расширить получаемый спектр поворотом кристалла на установке. Так, при $\theta_{p}=55.5^{\circ}$ (рис. $\left.3, b\right)$ наблюдается спектр сигнальной волны от 0.548 до $1.55 \mu \mathrm{m}$. Это позволяет решить поставленную прикладную задачу без необходимости менять местами детекторы.

Если угол оптической оси кристалла составляет $43^{\circ}$, спектр сигнальной волны будет изменяться от 0.5 до $0.81 \mu \mathrm{m}$, а спектр холостой волны от 0.81 до $1.6 \mu \mathrm{m}$, что полностью покрывает требуемый диапазон. Однако

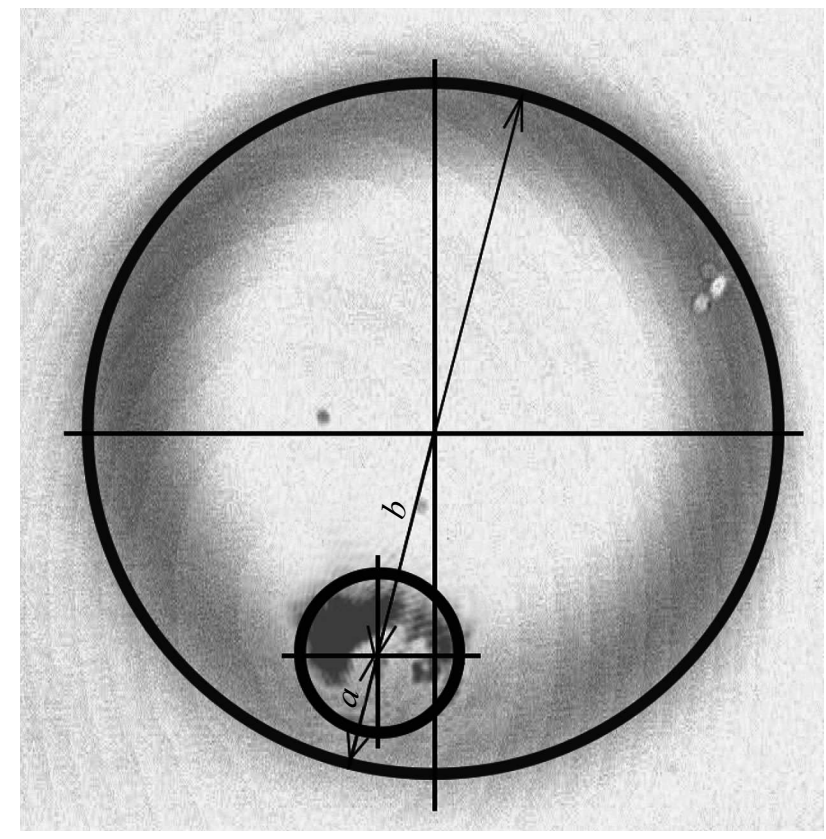

Рис. 5. Экспериментальное наблюдение явления СПР. Большое кольцо соответствует излучению сигнальной волны, малое - излучению накачки.

при этом для облучения одного детектора всем спектром фотонов необходимо будет переставлять его из одного канала в другой. Тем не менее, такая конфигурация все же имеет преимущество перед предыдущей угловой спектр генерируемых фотонов оказывается у́же, что упрощает конструкцию экспериментальной установки. Результат расчета для угла $\theta_{p}=43^{\circ}$ показан на рис. 4.

\section{Экспериментальная проверка результатов расчета}

На основе полученных результатов расчетов в дальнейшем будет создана система генерации бифотонного излучения с перестраиваемым спектром. На текущем этапе для проверки правильности результатов проведенных вычислений была собрана экспериментальная установка на основе имеющегося кристалла ВВО, полупроводникового лазера и USB-видеокамеры видимого диапазона. Цель эксперимента - использовав одно из частных решений, полученных выше, определить угол оптической оси кристалла и сравнить с его паспортными данными, тем самым проверив правильность вычислений.

По изображению СПР, полученному с помощью USBвидеокамеры на заданной длине волны, рассчитывалось значение в пикселях бо́льшего и меньшего отрезков, которые отсчитывались от центра луча накачки, как 


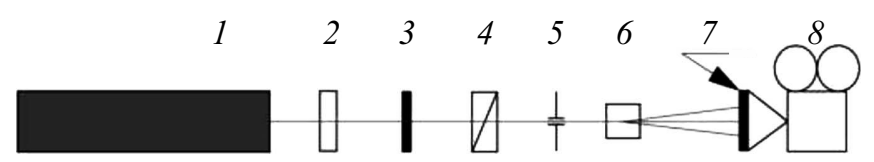

Рис. 6. Оптическая схема установки детектирования СПР: 1 - полупроводниковый лазер, $2-$ фазовая пластинка $\lambda / 2$, 3 - полосовой фильтр FB405-10, Thorlabs Inc., 4 - призма Глана-Томсона, 5 - лепестковая диафрагма, 6 - кристалл ВВО с углом оптической оси $35.4^{\circ}, 7$ - полосовой светофильтр FB640-10, Thorlabs Inc., 8 - камера видеоскан ВИДЕОСКАН-2-205.

показано на рис. 5. Затем вычислялось отношение

$$
r=a / b
$$

где $a$ - меньшая сторона в пикселях; $b-$ большая сторона в пикселях. Величины $a$ и $b$ пропорциональны синусам углов $\theta_{s 1}$ и $\theta_{s 2}$, которые получаются в результате расчета. Использование безразмерной величины $r$ вместо размерных $\theta_{s 1,2}$ избавляет от необходимости проводить абсолютные геометрические измерения, которые могут быть источниками дополнительных неопределенностей. Отношение $r$ является функцией $\theta_{p}$. Таким образом, сравнивая полученную в эксперименте величину $r$ и результаты расчета для различных $\theta_{p}$, можно определить угол оптической оси кристалла.

Для проверки результатов расчета использовалась оптическая установка, показанная на рис. 6, в которой источником накачки являлся полупроводниковый постоянный диодный лазер с длиной волны $405 \mathrm{~nm}$ и мощностью $10 \mathrm{~mW}$.

Оптическое излучение проходит через фазовую пластинку $\lambda / 2$ и призму Глана-Томсона 4 , которые позволяют задавать нужную плоскость поляризации. Призма Глана-Томпсона была выбрана потому, что по сравнению с обычным поляризатором не создает паразитной засветки из-за флуоресценции. Затем при помощи интерференционного фильтра 3 происходит селекция спектра оптического излучения в полосе от 400 до $410 \mathrm{~nm}$. На входе в кристалл ВВО 6 располагается лепестковая диафрагма 5, которая выделяет из всей диаграммы направленности лазера узкий луч и защищает от боковых засветок. Согласно спецификациям производителя, кристалл ВВО вырезан под углом оптической оси $35.4^{\circ}$. Излучение СПР на выходе из кристалла детектируется при помощи камеры ВИДЕОСКАН-2-205 8. На входе камеры установлен полосовой интерференционный фильтр 7 с полосой пропускания от 635 до $645 \mathrm{~nm}$. Данный фильтр вырезает заданную длину волны из спектра СПР и блокирует луч накачки. Для получения изображения луча накачки лазер был ослаблен при помощи нейтральных фильтров, таким образом, чтобы не оставлять переэкспонирования на матрице, а полосовой фильтр 7 был убран. Совмещенное изображение излучения СПР и луча накачки приведены на рис. 5.
Анализ изображения рис. 5, проведенный описанным выше методом, дал величину $r=0.18$. Такое значение $r$ соответствует $\theta_{p}=35.15^{\circ}$, что с погрешностью $0.7 \%$ согласуется с паспортными данными кристалла $-35.4^{\circ}$. Таким образом, разработанный алгоритм позволяет делать расчеты, хорошо согласующиеся с результатами эксперимента.

\section{Заключение}

Разработан конструктивный алгоритм определения параметров СПР в одноосных кристаллах. С его помощью рассчитана зависимость спектра СПР от угла оптической оси кристалла $\theta_{p}$ в диапазоне длин волн от 0.548 до $1.55 \mu \mathrm{m}$ при длине волны накачки $0.405 \mu \mathrm{m}$. Полученная зависимость позволила определить, что перекрытие всего рассматриваемого диапазона длин волн возможно. Угол оптической оси кристалла при этом должен быть $43^{\circ}$ для схемы с перестановкой детекторов из канала в канал и $55.5^{\circ}$ для схемы без перестановки. Также на основе сделанных расчетов был экспериментально определен угол оптической оси имеющегося кристалла. Измеренный угол хорошо совпал со спецификациями производителя, что подтверждает работоспособность метода. Данный метод и расчеты, полученные с его помощью, лягут в основу разрабатываемой установки для определения квантовой эффективности детекторов единичных фотонов по схеме Клышко-Пенина [7].

Авторы работы выражают благодарность старшему научному сотруднику А.И. Глазову и инженерам Я.А. Авдеевой и М.А. Солодиловой Всероссийского научноисследовательского института оптико-физических измерений за помощь в подготовке статьи.

Работа выполнена с использованием оборудования ЦКП высокоточных измерительных технологий в области фотоники (ckp.vniiofi.ru), созданного на базе ФГУП „ВНИИОФИ“ и поддержанного Минобрнауки России в рамках выполнения соглашения № 14.595.21.0003 от 28.08.2017 г. (уникальный идентификатор RFMEFI59517X0003).

\section{Список литературы}

[1] Gisin N., Ribordy G., Tittel W., Zbinden H. // Rev. Mod. Phys. 2002. V. 74. N 1. P. 145. doi 10.1103/RevModPhys. 74.145

[2] Электронный ресурс. Режим доступа: https://docbox.etsi.org/workshop/2014/201410_crypto/ /quantum_safe_whitepaper_1_0_0.pdf

[3] Ladd T.D., Jelezko F., Laflamme R., Nakamura Y., Monroe C., O'Brien J.L. // Nature. 2010. V. 464. N 4. P. 45. doi $10.1038 /$ nature 08812

[4] Электронный ресурс. Режим доступа: http://www.idquantique.com/

[5] Электронный ресурс. Режим доступа: http://www.magiqtech.com/ 
[6] Электронный ресурс. Режим доступа: https://www.idquantique.com/wordpress/wp-content/ /uploads/ID350-Datasheet.pdf

[7] Buller G. S., Harkins R. D., McCarthy A., Hiskett P.A., MacKinnon G.R., Smith G.R., Sung R., Wallace A.M., Lamb R.A., Ridley K.A., Rarity J.G. // Rev. Sci. Instrum. 2005. V. 76. P. 083112-083112-7.

[8] Turner G.K. // Bioluminescence and Chemiluminescence: Instruments and Applications / Ed. by Van Dyke K. V. 43-78. Boca Raton, FL: CRC Press, 1985.

[9] Rezus Y.L. A., Walt S.G., Lettow R., Renn A., Zumofen G., Götzinger S., Sandoghdar V. // Phys. Rev. Lett. 2012. V. 108. P. 093601.

[10] Eisaman M.D., Fan J., Migdall A., Polyakov S.V. // Rev. Sci. Instrum. 2011. V. 82. N 7. P. 071101. doi 0.1063/1.3610677

[11] Клышко Д.Н., Пенин А.Н. // УФН. 1987. Т. 152. № 4. С. 653.

[12] Shigeki Takeuchi // Jpn. J. Appl. Phys. 2014. V. 53. P. 030101.

[13] Магницкий С.А., Фроловцев Д.Н., Агапов Д.П., Демени А.В., Крутиков В.Н. // Измерительная техника. 2017. № 3. C. 24.

[14] Ginzburg V.M., Keratishvili N., Korzhenevich E.L., Lunev G.V., Penin A.N., Sapritsky V. // Opt. Eng. 1993. V. 32. N 11. P. 2911.

[15] Калачёв А.А. Спонтанное параметрическое рассеяние и задачи квантовой информатики/Учебно-методическое пособие. Казань: Казанский университет, 2012. 45 с.

[16] Boeuf N., Branning D.A., Chaperot I., Dauler E., Guerin S., Jaeger G.S., Muller A., Migdall A.L. // Opt. Eng. 2000. V. 39. N 4. P. 1016.

[17] Kato K. // IEEE J. Quantum Electron. 1986. V. 22. N 7. P. 1017. doi 10.1109/JQE.1986.1073097 